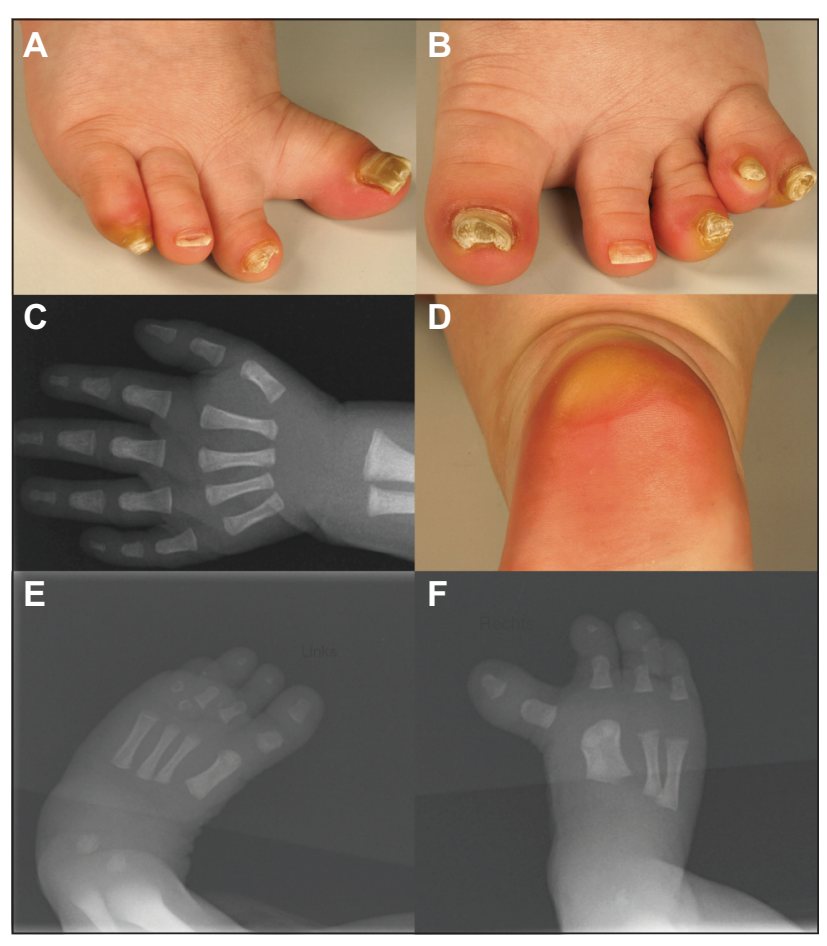

Figure 1. A, B) Clinical phenotype of the feet (A, right foot; $\mathrm{B}$, left foot); C) radiograph of the normal left hand; D) subungual hyperkeratosis and keratoderma of the sole consistent with a diagnosis of pachyonychia type I; E, F) skeletal radiographs showing unclassifiable dysplasia.

Gaithersburg, MD, USA) and demonstrated a known heterozygous trinucleotide deletion c.389 391delCCT (causing p.Ser130del) in exon 1 of the $K R \bar{T} 16$ gene.

The combination of pachyonychia congenita type I and the above-mentioned unusual skeletal malformation has not been previously described. It is tempting to speculate that the abnormalities are not coincidental. As it seems biologically implausible that a KRT16 mutation could affect skeletal development, one might assume the existence of a contiguous gene syndrome. However, that is unlikely as we found a trinucleotide deletion in the KRT16 gene for which the patient is clearly heterozygous, thus ruling out microdeletions encompassing all or part of KRT16. It is therefore more prudent to assume that there is no causal relationship. It is even debatable whether the skeletal abnormality is genetically determined, as it cannot be classified into one of the known categories of acral developmental abnormalities. Thus, we think that the concurrence we describe is a coincidental one. It is, however, quite possible that the abnormal skeleton can contribute to the pachyonychia and concomitant keratoderma by altering pressure distribution.

We also suggest that the other skeletal defects that have been reported in association with pachyonychia type I were not causally related. The relatively high frequency in the literature may be due to an ascertainment bias.

Acknowledgements. Mutation analysis was made possible by the International Pachyonychia Congenita Consortium. MvS is supported by the University Hospital Maastricht, the GROW research institute for oncology and

developmental biology, the Netherlands institute for scientific research (ZONMW) grant 907-00-202.

Department of Dermatology,

Maastricht University Medical

Center+, P. Debijelaan 25, 6202AZ

Maastricht, the Netherlands

$<$ mauricevansteensel@gmail.com>

1. Leachman SA, Kaspar RL, Fleckman P, et al. Clinical and pathological features of pachyonychia congenita. J Investig Dermatol Symp Proc 2005; 10: 3-17.

2. Connors JB, Rahil AK, Smith FJ, Mclean WH, Milstone LM. Delayed-onset pachyonychia congenita associated with a novel mutation in the central 2B domain of keratin 16. Br J Dermatol 2001; 144: 1058-62.

3. Murugesh SB, Reddy S, Ragunatha S, Mohammed Faizal MM, Shashikala P. Acro-osteolysis: a complication of JadassohnLewandowsky syndrome. Int J Dermatol 2007; 46: 202-5.

doi: $10.1684 /$ ejd. 2009.0643

\section{Syphilis surveillance in Portugal, 2000-2005}

The annual incidence of acquired syphilis in developed countries dropped to very low levels in the early 1990s, partly due to aggressive HIV campaigns that emphasized condom use. However, a series of syphilis outbreaks have been reported in several northern and western European countries since 1996. These outbreaks have disproportionately involved men who have sex with men (MSM), people who engage in high-risk sexual behavior (e.g., unprotected sex with prostitutes or multiple partners), and people who use new recreational drugs [1]. The purpose of this paper is to analyze the syphilis trends from 2000 to 2005 in Portugal based on data collected by the Sentinel Network for Sexually Transmitted Infections (STIs) Surveillance.

Operating since 2000, the Sentinel Network System was established to collect data on all STIs in Portugal. It involves eight public hospitals and health-center based STI clinics that cover about $70 \%$ of the Portuguese population, relies on voluntary participating dermatovenereologists and receives self-referred and physician-referred patients. From 2000 to 2005, 5,020 cases of Sexually Transmitted Infections were reported by this Sentinel Network System and Syphilis testing was routinely offered to all patients.

The organization aims to determine STI incidence trends and to monitor epidemiological profiles of STI patients. Syphilis notifications contain information on gender, residence, sexual orientation, country of birth, HIV status, educational level attained, number of sexual partners during the last six months, intravenous drug and condom use habits. Data security and strict patient confidentiality are always maintained. The data presented in this paper include infectious syphilis cases (primary, secondary and early latent) reported between January 2000 and December 2005. Statistical analyses were performed with SPSS ${ }^{\circledR}$ v.13.0. Proportions were compared using the Chi-square test.

From 2000 to 2005, 784 cases of recent syphilis were reported in Portugal. Specifically, these include 208 


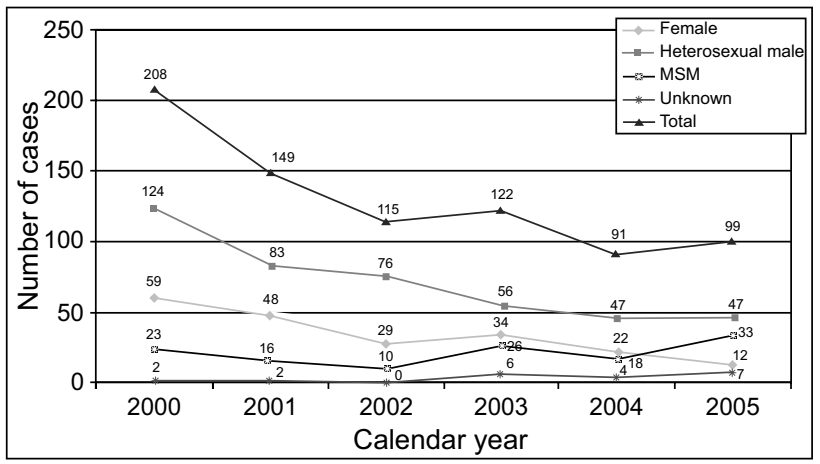

Figure 1. Evolution of the number of syphilis patients by gender/sexual orientation.

reported in 2000, 149 in 2001, 115 in 2002, 122 in 2003, 91 in 2004 and 99 in 2005. Although the increase from 91 to 99 cases from 2004 to 2005 was not statistically significant, it is worth noting that it was linked to a syphilis outbreak that occurred in 2005 among MSM. Indeed, from 2000 and 2005, the proportion of syphilis cases involving MSM increased ( $\mathrm{p}<0.001)$, while that involving females and heterosexual men decreased and remained stable, respectively (figure 1). Of the 767 syphilis cases with HIV status data, 136 (17.3\%) were HIV-positive, including 51 patients who received their HIV and syphilis diagnoses concurrently and 85 who had previously been diagnosed as HIV-positive. HIV prevalence was $43.3 \%$ in MSM, $11.3 \%$ in heterosexual men, and $10.8 \%$ in females. There was a general increase in the number of HIVpositive patients in Portugal after $2002(\mathrm{p}<0.001)$.

The Sentinel Network data indicate a selective increase in syphilis infections in MSM since 2000, while the number of heterosexually transmitted cases remained relatively stable. However, the proportion of heterosexually transmitted cases was relatively large for Western Europe. The frequent occurrence of syphilis and HIV coinfection is of great concern; these patients are also at risk for gonorrhea, chlamydia infections and L2 chlamydia proctitis [2-4]. Media coverage, syphilisawareness advertisements in the gay media and distribution of literature to the homosexual community are evidently insufficient to contain syphilis spreading among MSM in Portugal.

In conclusion, there has been a surge in syphilis rates in many Western countries since the late 1990s. However, this trend only emerged in Portugal the past few years. This review of syphilis cases between 2000 and 2005 shows similar findings to epidemiological trends in other European countries. Syphilis screening should be emphasized for MSM, especially for those being treated for HIV.

Acknowledgements. Financial support: none. Conflict of interst: none.

GEIDST, Hospital Curry Cabral, Rua da Beneficência $N^{\circ} 8,1069$ 639 Lisboa, Portugal <ricardolaocoelho@yahoo.com>
1. Fenton KA. Recent trends in the Epidemiology of Sexually. Transmitted Infections in the European Union. Sex Transm Infect 2004; 80: 255-63.

2. Nicoll A, Hamers $F$. Are trends in HIV, gonorrhoea and syphilis worsening in western Europe? BM 2002; 324: 1324-7.

3. Zetola N, Klausner J. Syphilis and HIV infection: An Update. Clin Infect Dis 2007; 44: 1222-8.

4. Stary A, Stingl G. Sexually transmitted infections. Eur J Dermatol 2007; 17: 107-8.

doi:10.1684/ejd.2009.0646

\section{Contact dermatitis from an anti- haemorroidal cream containing ruscogenin}

Ruscus aculeatus L. is a member of the Liliaceae family and is native to Mediterranean Europe and Africa. It has been widely used as a laxative and diuretic agent and as a vasoconstrictor in the topical treatment of varices and haemorrhoids. The pharmacological activity of Ruscus aculeatus $L$. is attributed to steroidal saponins, mainly ruscogenin (figure 1) and neoruscogenin, which have venoconstricting and antiinflammatory effects $[1,2]$.

A 35-year-old man presented with pruritic erythematous lesions on the perianal area and buttocks 5 days after initiating the local application of Ruscus Llorens ${ }^{\circledR}$ (Llorens, Barcelona, Spain) cream for haemorrhoids. The lesions became papulo-erythematous, well-defined and spread within a day to the trunk and both legs.

The patient had used other antihaemorrhoidal creams previously with no cutaneous reactions. Therapy with the cream was stopped and the patient was successfully treated with oral corticosteroids.

The antihaemorrhoidal cream contained ruscogenin, cinchocaine hydrochloride (dibucaine), prednisolone, menthol, zinc oxide and other excipients in its composition. The patient was patch tested with GEIDAC (Spanish Group of Investigation of Contact Dermatitis) standard series, local anaesthetics series and Ruscus llorens ${ }^{\circledR}$ cream as is. Caine mix, tixocortol pivalate, Ruscus llorens ${ }^{\circledR}$ cream, and cinchocaine patch tests were positive $(++)$ at 48 and 96 hours, and tetracaine $(++)$ and lidocaine $(+)$ at 96 hours.

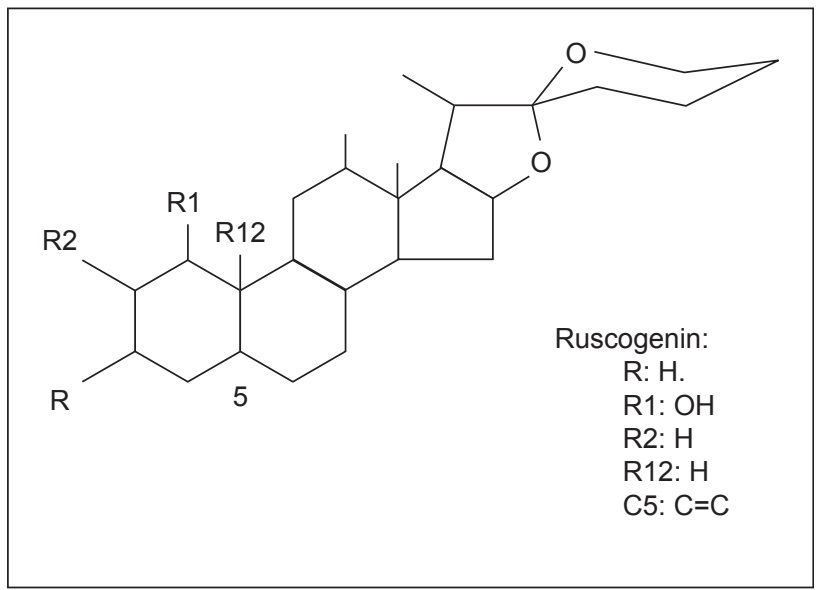

Figure 1. Chemical structure of ruscogenin. 